\title{
Computer Solution of the Sun Shadow Positioning Model
}

\author{
Wenyan Dong \\ School of Electrical \& Electronic Engineering, North China Electric Power University, Baoding \\ 071003, China; \\ dongwenyan666@163.com
}

Keywords: Least square method, Curve fitting, Exhaustive method.

\begin{abstract}
This paper mainly uses the angle of declination angle, and solar elevation angle and azimuth of the sun between the relationship, according to them with the longitude and latitude, date and time of the conversion formula, and the use of MATLAB software were calculated. It is concluded that the effects of long three parameters are: the latitude and longitude, date and time was established, and the eventual establishment of sun shadow location model. And use the model to solve problems in various practical problems. Using coordinate to calculate change affects the length of time, then the least square method for curve fitting, draw the local noon corresponding to the Beijing time, and use the time difference formula to calculate the longitude of the straight rod is located, again according to the azimuth of the sun and shadow coordinates and the his angle of multiple relations, the use of MATLAB to solve the latitude, final position according to Google map out of place.
\end{abstract}

\section{Introduction}

How to determine the video was filmed on location and shooting date is an important aspect of video data analysis, sun shadow positioning technology is through solar shadow variation of objects in video analysis, a method to determine the video was filmed on the place and date. The mathematical model of the shadow length change is established, and the variation law of the shadow length is analyzed, and the change of the length of the solar shadow is drawn by the model. A mathematical model is established to determine the location and date of the straight rod according to the data of the solar shadow vertex coordinates of a fixed straight bar on the horizontal ground.

\section{Analysis}

According to the Internet to find information, we conclude that the shadow length can be calculated by the length of the straight rod itself and the solar altitude angle, and solar elevation angle and the straight rod position (longitude and latitude), measurements of the date and time related, so you can get influenced long by a factor of three. Based on the knowledge of geography, we learned that day film shortest time is the noon local time and can be obtained shadow length on each parameter variation, the length of the shadow change model is established. Then in MATLAB can be obtained by curve fitting the shadow length corresponding to the short Beijing time, and then use the knowledge of geography can be obtained by measuring the longitude of the location. According to the longitude can derive a declination angle and according to the relationship between the azimuth of the sun and shadow coordinates, and it on several parameters in the model formula, you can get solar azimuth angle and the latitude of the relational model, using MATLAB can be obtained latitude in a range of values. According to the latitude and longitude range, it can be concluded that a number of possible straight.

\section{Assumptions}

1. The effect of the refractive index of air on the shadow is very small, and it can be ignored.

2. All the data we get are true and reliable. 
3. The ground is projected to the absolute level of a straight bar.

4. The wind is weak, and the effect of the rod is negligible.

5. The pole as approximate straight line segments, and perpendicular to the ground.

6. Measurement error is ignored.

\section{Symbol Definitions}

\begin{tabular}{cl}
\hline Symbols & Definitions \\
\hline $\mathrm{x}$ & Horizontal coordinates of shadow vertices \\
$y$ & The vertical coordinates of the shadow vertices \\
$\theta$ & Geographical longitude \\
$\varphi$ & Geographical latitude \\
$\delta$ & Solar Declination Angle \\
$\mathrm{h}$ & Solar zenith angle \\
$\mathrm{A}$ & Solar azimuth \\
$\mathrm{t}$ & Time angle \\
$\mathrm{n}$ & Beijing time \\
$\mathrm{N}$ & From New Year's day to the total number of days \\
$\mathrm{H}$ & Rod length \\
$\mathrm{L}$ & The length of the shadow \\
\hline
\end{tabular}

\section{The Rod Shadow Change Model}

The shadow length change model is set up, according to the geographical knowledge, we know that the length of the shadow basic position decided by the sun. And the sun position is mainly composed of local latitude, season, year, month, day) and the three factors, and can use the latitude, solar declination Angle, Angle of the sun, the parameters such as Angle position. We reviewed the current domestic most of the sunshine analysis software used to adopt the relevant content in literature [1], including the basic concept of architectural sunshine and design program, etc., it is concluded that the main calculation method of each parameter and the sun position:

The solar declination angle $\delta$ :The angle between the center of the earth and the sun at the center of the line and the equatorial plane of the earth is the solar declination angle. It is in the vernal equinox and autumnal equinox time is equal to zero, and in extreme summer solstice and winter solstice, respectively $\pm 23^{\circ} 26^{\prime} 30$ " , . The formula is as follows:

$\delta=23.45^{\circ} \times \sin [(\mathrm{N}-80.25) \times(1-\mathrm{N} / 9500)]$

Time angle t: Unit time of the earth's rotation Angle is Angle. Noon hour Angle of $0^{\circ}$, earth rotation a week $360^{\circ}$, the corresponding time for 24 hours, per hour when the Angle is $15^{\circ}$, Angle of $1^{\circ}$ when every four minutes. Again, due to the time of Beijing is, in fact, $120^{\circ} \mathrm{E}$, therefore, its computation formula is as follows:

$$
\mathrm{t}=15^{\circ} \times\left[\left(\theta-120^{\circ}\right) \times \frac{1}{15}+\mathrm{n}-12\right]
$$

Solar azimuth A:The rays of the sun on the ground plane projection and local meridian angle, which can be approximated by was erected angle of straight lines on the ground in the sun shadow and south. The formula is as follows:

$$
\sin A=\frac{\cos \delta \times \sin t}{\cos h}
$$

Solar zenith angle h: The angle between the line of a point on the earth's surface and the sun's connection to the horizon, and the maximum height of the sun at noon . The formula is as follows:

$\mathrm{h}=\arcsin (\sin \varphi \times \sin \delta+\cos \varphi \times \cos \delta \times \cos \mathrm{t}),-90^{\circ} \leq \mathrm{h} \leq 90^{\circ}$

Finally, the formula for calculating the length of the shadow can be obtained.

$\mathrm{L}=\mathrm{H} \times \cot \mathrm{h}$ 


\section{The Least Square Curve Fitting Method}

For the fitting problem, if the basis function is based on the basis of the basis function as an extension of the algebraic space,it can be given a simple explanation of the least squares.

For a given set of data $\left\{\left(\mathrm{x}_{\mathrm{i}} \mathrm{y}_{\mathrm{i}}\right)\right\}_{\mathrm{i}=1}^{\mathrm{m}}$, Assumes that the fitting function:

$\varphi(\mathrm{x})=\alpha_{0} \varphi_{0}(\mathrm{x})+\alpha_{1} \varphi_{1}(\mathrm{x})+\cdots+\alpha_{\mathrm{n}} \varphi_{\mathrm{n}}(\mathrm{x})$

The basis function is the function of linearly independent department. Least squares fitting problem is coefficient, make the following expression to take minimum

$\mathrm{E}=\sum_{\mathrm{i}=1}^{\mathrm{m}}\left[\sum_{\mathrm{j}=0}^{\mathrm{n}} \alpha_{\mathrm{j}} \varphi_{\mathrm{j}}(\mathrm{x})-\mathrm{y}_{\mathrm{i}}\right]^{2}$

According to the extreme value problem of multivariate function, the necessary condition for $\mathrm{E}$ to take the extreme value is

$\frac{\partial \mathrm{E}\left(\alpha_{0}, \alpha_{1} \cdots \alpha_{n}\right)}{\partial \alpha_{i}}=0$

$\mathrm{a}=\left[\begin{array}{c}\alpha_{0} \\ \alpha_{1} \\ \vdots \\ \alpha_{\mathrm{n}}\end{array}\right], \quad \mathrm{y}=\left[\begin{array}{c}\mathrm{y}_{1} \\ \mathrm{y}_{2} \\ \vdots \\ \mathrm{y}_{\mathrm{m}}\end{array}\right]$

And $A=\left[\alpha_{i j}\right]_{m \times(n+1)}, \quad \alpha_{i j}=\varphi_{j}\left(x_{i j}\right)$,so:

$\mathrm{E}=\|\mathrm{Aa}-\mathrm{y}\|_{2}^{2}$

So least-square fitting problem was converted to least square solution of overdetermined linear equations :

$\mathrm{Aa}=\mathrm{y}$

Set of linear equations is the real number field $(m, n)$ order equation. The above equations are necessary and sufficient conditions of the solutions is the equation coefficient matrix and the rank of the augmented matrix is the same.

$\operatorname{rank}(\mathrm{A})=\operatorname{rank}([\mathrm{A}, \mathrm{y}])$

But sometimes equations do not meet the above conditions, so there is no general sense of the solution, such a set of equations called contradictory equations. If there is $\alpha_{s}$ :

$\mathrm{f}(\mathrm{x})=\|\mathrm{Aa}-\mathrm{y}\|_{2}$

The least squares solution of $\mathrm{a}=\mathrm{as}$ is the least, while as is the least square solution of the equation $\mathrm{Aa}=\mathrm{y}$. As is a necessary and sufficient condition for the least squares solution of the equations is that $\alpha_{s}$ is a solution of the equations.

$\mathrm{A}^{\mathrm{T}} \mathrm{A} \alpha=\mathrm{A}^{\mathrm{T}} \mathrm{y}$

The above all the letters from the formula does not have a practical meaning in life, just a mathematical sense.

According to a fixed straight rod at ground level the sun shadow vertex coordinate data, establish mathematical model to determine the location of the straight rod. According to the model, we can know the shadow length changes with time, and reaches the minimum value in the location time at noon. Therefore, we can use shadow vertex coordinate data shadow length corresponding to the different time is obtained, and in the MATLAB software using curve fitting method to find the lowest point of the curve and corresponding is corresponding to locations of noon Beijing time. According to the geographical knowledge, it can get the local longitude calculation formula:

$\theta=15^{\circ} \times(12-\mathrm{n})+120^{\circ}$

In addition, according to the definition of the azimuth of the sun and the projection theorem, the formula for calculating the azimuth angle is:

$$
\mathrm{A}=\arctan \frac{\mathrm{y}}{\mathrm{x}}
$$

When shooting date is unknown, using MATLAB processing video length of rod and a long shadow, the shadow length of time variation, fitting long shadow the most short time corresponds to the time, again by finding longitude method to calculate the longitude, known rod length and shadow long for latitude, declination angle, from and to determine the location of, by the angle of declination and the introduction of $\mathrm{N}$, calculate the date. 


\section{Summary}

In the model, when solving the longitude, we can use more accurate fitting method. In addition, the solution range can be increased by using the time angle and the latitude angle, so that the situation of the southern hemisphere can be obtained. In this paper, the location model of the sun's shadow can be established according to the change of building shadow to determine the date and place, and the various angles of the sun were studied. Can be applied to study the orientation of buildings, the movement of celestial bodies and other aspects of the study.

\section{References}

[1]. Jianting Chen. On the Calculation of the Optimum Solar Azimuth. JOURNAL OF ZHAOQING UNIVERSITY. Vol.35 (2014) No. 2, p. 20-22.

[2]. Guoan Wang. Calculation of the Change Range of the Sun High Angle and the Angle of Sunrise and Sunset in One Year. Meteorological and Environmental Sciences. Vol.30 (2007) No. 12, p. 161-164.

[3]. Zhijie Cai. Identify Geographical Location from Sun Shadow. Mathematical Modeling and Its Applications. Vol.4 (2015) No. 4, p. 25-33. 